\title{
Effect of bovine lactoferricin on DNA methyltransferase 1 levels in Jurkat T-leukemia cells
}

\author{
T.-N. Zhang and N. Liu ${ }^{1}$ \\ Key Laboratory of Dairy Science, Ministry of Education, College of Food Science, Northeast Agricultural University, 59 Mucai Street, \\ Xiangfang District, Harbin 150030, China
}

\begin{abstract}
Abnormal methylation of the promoter of several genes is common in patients with acute lymphoblastic leukemia. Methylation of DNA is brought about by DNA methyltransferases (DNMT). Bovine lactoferricin (Lfcin B) is a cationic peptide that possesses potent in vitro and in vivo anticancer activity and might affect the expression of DNMT1. In the current study, we determined the mRNA and protein expression of DNMT1 in Jurkat T-leukemia cells, after incubation with Lfcin B, by real-time quantitative reverse transcription PCR and Western blot analysis. The results of real-time quantitative reverse transcription PCR showed that DNMT1 expression in Jurkat T-leukemia cells was reduced after treatment with Lfcin B, and Lfcin B reduced the half-life of DNMT1 mRNA from approximately 8 to $2 \mathrm{~h}$. The results of Western blot analysis showed that the expression of DNMT1 protein was down-modulated by Lfcin B in Jurkat T-leukemia cells. Moreover, we found that protein biosynthesis in Jurkat T-leukemia cells was essential for Lfcin B to down-modulate the expression of DNMT1.
\end{abstract}

Key words: bovine lactoferricin, Jurkat T-leukemia cell, DNA methyltransferase, DNMT1 mRNA

\section{INTRODUCTION}

Lactoferricin B (Lfcin B) is a cationic antimicrobial peptide with cytotoxic activity against microorganisms and human cancer cells (Eliassen et al., 2002, 2006; Enrique et al., 2009). It consists of 25 amino acid residues (17 to 41 proximal to the $\mathrm{NH}_{2}$ terminus of bovine lactoferrin) and is produced by acid-pepsin hydrolysis of bovine lactoferrin (Bellamy et al., 1992a). Bovine lactoferrin is an $80-\mathrm{kDa}$ iron-binding glycoprotein found both in the secretory granules of neutrophils and in biological fluids, including saliva and milk (Lonnerdal and Iyer, 1995). Lactoferricin B is an antimicrobial peptide

Received December 21, 2009.

Accepted May 3, 2010.

${ }^{1}$ Corresponding author: ningliu6666@yahoo.com.cn that displays no iron-binding capacity (Bellamy et al., 1992b).

Substantial levels of Lfcin B are found in the human stomach following ingestion of bovine lactoferrin (Kuwata et al., 1998), indicating that Lfcin B is a natural breakdown product from the digestion of cow's milk. Lactoferricin $\mathrm{B}$ has attracted considerable interest because of its well-established antimicrobial activity (Tomita et al., 1991), and recent evidence indicates that Lfcin B possesses potent activity against cancer cells in vivo (Tsuda et al., 1998; Cho et al., 2004). Subcutaneous administration of Lfcin B to mice inoculated with L5178Y-ML25 murine lymphoma cells or B16-BL6 murine melanoma cells resulted in a significant inhibition of liver and lung metastases (Yoo et al., 1997). Although the molecular basis of the relationship between Lfcin B and methylation of Jurkat T-leukemia cells is not yet clear, one possibility is that the cationic, amphiphatic nature of Lfcin B could affect the expression of DNA methyltransferases (DNMT) and stability of DNMT mRNA in Jurkat T-leukemia cells (Vogel et al., 2002).

Methylation of DNA plays a significant role in the tissue- and stage-specific modulation of genes (Kass et al., 1997; Singal and Ginder, 1999), genomic imprinting (Nakao and Sasaki, 1996; Bartolomei and Tilghman, 1997), and X-chromosome inactivation (Panning and Jaenisch, 1998) and has been shown to be essential for normal mammalian development ( $\mathrm{Li}$ et al., 1992). The DNMT known to date are DNMT1, DNMT1b, DNMT1o, DNMT1p, DNMT2, DNMT3A, DNMT3b with its isoforms, and DNMT3L (Robertson, 2002). Mizuno et al. (2001) observed that DNMT1, DNMT3A, and DNMT3b were increased 5.3-, 4.4- and 11.7-fold, respectively, in acute myelogenous leukemia compared with control bone marrow cells. Their study also demonstrated a more modest but significant increase in these 3 DNMT in the acute phase of chronic myelogenous leukemia.

Expression of DNMT is elevated in leukemia and aberrant methylation is common, with a decrease in the total genomic content of 5-methylcytosine and concomitant hypermethylation of $\mathrm{CpG}$ island-associated tumor suppressor genes. Aberrant DNA hypermethylation is 
thought to be involved in leukemogenesis. For example, aberrant hypermethylation of the $p 15^{I N A K 4 B}$ tumor suppressor gene is associated with its inactivation in at least half of patients suffering from acute lymphoblastic leukemia and myelogenous leukemia (Herman et al., 1996, 1997).

It has recently been reported that modulation of ceramide metabolism in T-leukemia cell lines potentiates apoptosis induced by bovine lactoferricin (Furlong et al., 2008). A link between Lfcin B and DNMT1 expression in Jurkat T-leukemia cells has not been established. We investigated the effects of Lfcin B on the downmodulation of DNMT1 expression and the stability of DNMT1 mRNA in Jurkat T-leukemia cells.

\section{MATERIALS AND METHODS}

\section{Cell Culture and Lfcin B Treatment}

Jurkat T-leukemia cell clone E6-1 was obtained from Cell Bank, Chinese Academy of Sciences (Shanghai). Cell lines were maintained at $37^{\circ} \mathrm{C}$ in a $5 \%$ or $10 \%$ $\mathrm{CO}_{2}$ humidified atmosphere in RPMI-1640 medium (HyClone Laboratories, Logan, UT) containing 5\% heat-inactivated fetal bovine serum, $2 \mathrm{mM}$ glutamine, $100 \mathrm{mg} / \mathrm{mL}$ streptomycin, and $100 \mathrm{U} / \mathrm{mL}$ penicillin. Jurkat T-leukemia cells (4 passages) were suspended at a density of $5 \times 10^{5}$ cells $/ \mathrm{mL}$ in culture medium and incubated for $0,6,12,18$, and $36 \mathrm{~h}$ in the presence of Lfcin B at concentrations of 0, 50, 100, and $200 \mu \mathrm{g} / \mathrm{mL}$. Lactoferricin B (amino acid sequence: FKCRRWQWRMKKLGAPSITCVRRAF) derivatives were synthesized in linear form by Sangon (Shanghai, China).

\section{Actinomycin D Experiment}

Cells were grown for $4 \mathrm{~h}$ in the absence and presence of Lfcin B $(200 \mu \mathrm{g} / \mathrm{mL})$ and blocked with $5 \mu \mathrm{g} /$ $\mathrm{mL}$ actinomycin $\mathrm{D}$ (an mRNA synthesis inhibitor). Cells were collected at $0,2,4$, and $8 \mathrm{~h}$ after treatment with actinomycin D. Total RNA was isolated, treated with DNase I, and reverse-transcribed into cDNA. The level of DNMT1 transcript was measured by real-time quantitative reverse transcription PCR (qRT-PCR) analysis.

\section{Total RNA Extraction and qRT-PCR}

Cultured cells were washed twice with PBS and harvested. Total RNA from cells was isolated using the MagMAX-96 Total RNA Isolation Kit (ABI-Ambion, Austin, TX) according to the manufacturer's instructions. The concentration and purity of the total RNA samples were assessed using the DU $800 \mathrm{UV} /$ Visible Spectrophotometer (Beckman Coulter, Fullerton, CA). Synthesis of cDNA from RNA was performed with a reverse transcription system kit (Promega, Madison, WI) according to the manufacturer's instructions. Real-time quantitative PCR was carried out in 96-well polypropylene microplates on an ABI Prism 7500 (Applied Biosystems, Foster City, CA) using SYBR Green Real-time PCR Master Mix (Toyobo, Tokyo, Japan) according to the manufacturer's instructions.

Primers for DNMT1 were: forward 5'-CTTCTTCAGCACAACCGTCA-3'; reverse 5'-GAA GAG CCGGTAGGTGTCAG-3'. Primers for $\beta$-actin were: forward 5'-GCAGATGTGGATCAGC AAGC-3'; reverse 5'-ATAAAGCCATGCCAATCTCATC-3'. Primers for proliferating cell nuclear antigen (PCNA) were: forward 5'-AGGAAGCTGTTACCATAGAGA-3'; reverse 5'-ACAACAAGGGGTACATCTGC-3'. Realtime quantitative $\mathrm{PCR}$ was performed in a $20-\mu \mathrm{L}$ reaction mixture prepared with a real-time PCR Master Mix kit containing a diluted cDNA solution, $10 \mu M$ of each primer, and $10 \mu \mathrm{L}$ of SYBR Green real-time PCR Master Mix under the following conditions: 1 cycle at $95^{\circ} \mathrm{C}$ for $5 \mathrm{~min}, 40$ cycles at $95^{\circ} \mathrm{C}$ for $10 \mathrm{~s}$, and at $60^{\circ} \mathrm{C}$ for $40 \mathrm{~s}$.

Target cDNA was quantified using a relative quantification method. The quantity of DNMT1 transcript in each sample was standardized to $\beta$-actin or PCNA transcript levels. The data analyses were performed according to the $2^{-\Delta \Delta \mathrm{CT}}$ method introduced previously (Livak and Schmittgen, 2001).

\section{Western Blot Analyses}

Goat anti-DNMT1 antibody, mouse anti-goat horseradish peroxidase (HRP)-conjugated second antibody, mouse anti- $\beta$-actin antibody, and goat anti-mouse HRP-conjugated second antibody were purchased form Santa Cruz Biotechnology (Santa Cruz, CA). Jurkat T-leukemia cells in complete RPMI-1640 medium containing $5 \%$ heat-inactivated fetal bovine serum were cultured in the presence of Lfcin B (at 0, 50, 100, and $200 \mu \mathrm{g} / \mathrm{mL}$ ) for $6,12,18$, and $36 \mathrm{~h}$. Cells were lysed in protein lysis buffer containing $7 \mathrm{~mol} / \mathrm{L}$ of urea, 2 $\mathrm{mol} / \mathrm{L}$ of thiourea, $4 \%$ CHAPS (3-[(3-cholamidopropyl)dimethylammonio]-1-propane sulfonate), $65 \mathrm{mmol} / \mathrm{L}$ of dithiothreitol, 0.2\% (pH 3-10) Bio-Lyte (Bio-Rad, Hercules, CA), and complete protease cocktail inhibitors (Roche, Laval, Canada). Lysates were ultrasonicated and cleared by centrifugation at $13,000 \times g$ at $4^{\circ} \mathrm{C}$ for 10 min, and the supernatant was collected. Proteins were quantified by using the Bradford method. Samples were boiled in SDS sample buffer and total protein $(20 \mu \mathrm{g})$ was loaded into each well of an $8 \%$ SDS-polyacrylamide 


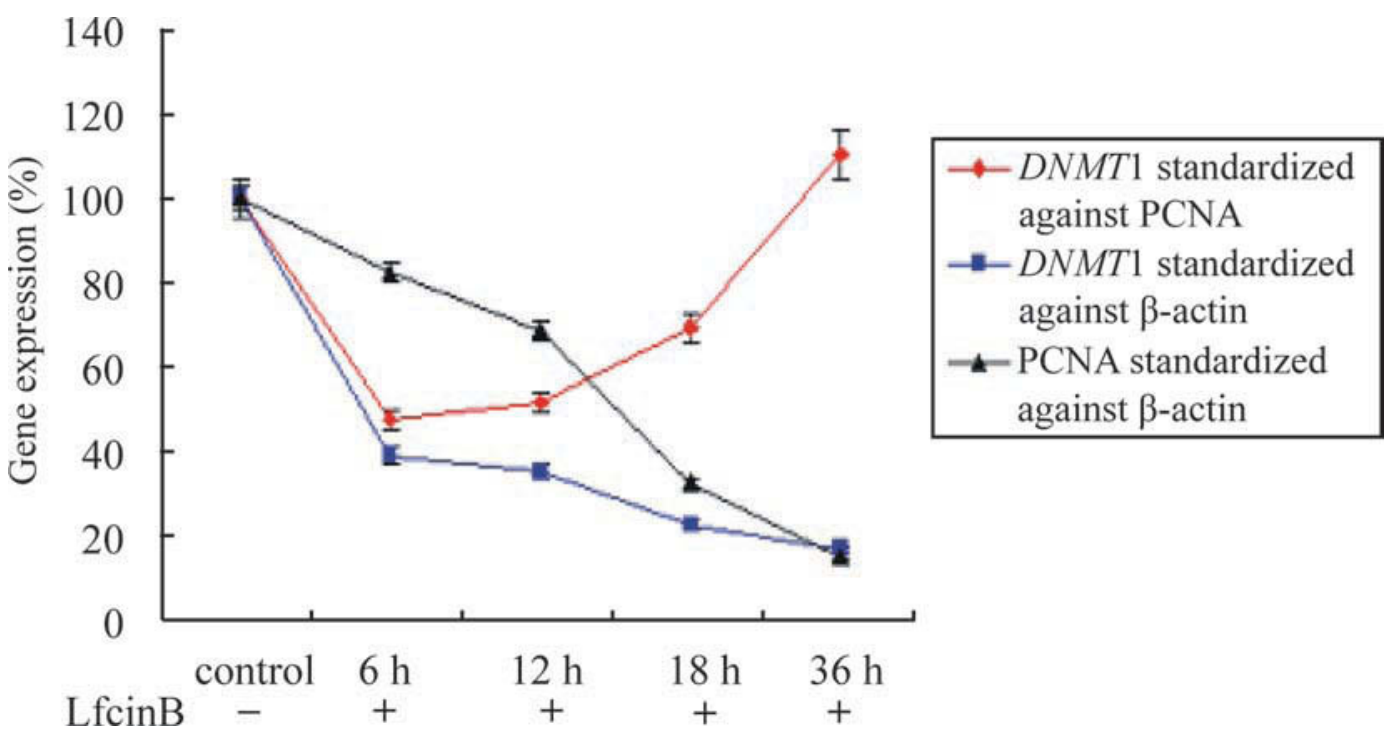

Figure 1. Down-modulation of DNA methyltransferase (DNMT)1 mRNA expression in Jurkat T-leukemia cells by lactoferricin B (Lfcin B). Expression of mRNA was quantified by real-time reverse transcription PCR. Cells were incubated for $0,6,12,18$, and $36 \mathrm{~h}$ in the presence or absence of Lfcin B at a concentration of $200 \mu \mathrm{g} / \mathrm{mL}$; the control was incubated for $36 \mathrm{~h}$ without Lfcin B. PCNA = proliferating cell nuclear antigen. PCR results were expressed as percentage of their respective controls. Three samples were measured for each group and results represent means $\pm \mathrm{SE}$ of the 3 groups. Color version available in online PDF.

gel for separation by electrophoresis. Protein bands were transferred onto nitrocellulose membranes. The resulting blots were blocked overnight with PBS-Tween 20 (0.25 mol/L Tris, $150 \mathrm{mmol} / \mathrm{L} \mathrm{NaCl}, 0.2 \%$ Tween 20 in PBS) containing $3 \%$ powdered skim milk and then probed overnight with the desired goat anti-DNMT1 antibody primary antibody at a 1:500 dilution. Blots were then washed with PBS-Tween 20 and probed for $1 \mathrm{~h}$ with HRP-conjugated mouse anti-goat second antibody $(1: 10,000)$ as appropriate. Following additional washes with PBS-Tween 20, the protein bands were visualized using an enhanced chemiluminescence detection system (GE Healthcare, Piscataway, NJ).

\section{Statistical Analysis}

Statistical analysis was performed using Statistical Program for Social Sciences (SPSS) software 13.0 (SPSS Inc., Chicago, IL). Data were analyzed by ANOVA to identify significant $(P<0.05)$ differences between the groups. All experimental groups that met the initial ANOVA criteria, were compared using post hoc Bonferroni $t$-tests, with the assumption of 2-tailed distribution and 2 samples with equal variance at the $P<0.05$ level. Statistical significance is marked by asterisks in the figures.

\section{RESULTS}

\section{Lfcin B Down-Modulates the Level of DNMT1 mRNA in Jurkat T-Leukemia Cells}

Jurkat T-leukemia cells were incubated in the presence or absence of Lfcin B at a concentration of $200 \mu \mathrm{g} /$
$\mathrm{mL}$ for $6,12,18$, or $36 \mathrm{~h}$. After incubation, total RNA was isolated and treated with DNase I, quantified, and reverse-transcribed into cDNA. The DNMT1 transcript levels were measured by qRT-PCR analysis of cDNA. The results were standardized to $\beta$-actin and PCNA cDNA levels. The PCNA level was also standardized against $\beta$-actin cDNA.

Treatment with Lfcin B for $36 \mathrm{~h}$ reduced the level of NDMT1 mRNA by nearly $85 \%$ in Jurkat T cells. The results show DNMT1 transcript levels standardized against $\beta$-actin transcript (Figure 1). In addition, Figure 1 showed that Lfcin B down-modulated the expression of PCNA, and thus might arrest the cell cycle to some extent, which was consistent with the conclusion of Freiburghaus et al. (2009).

\section{Lfcin B Down-Modulates DNMT1 Levels in Jurkat T-Leukemia Cells}

Jurkat T-leukemia cells were incubated in the presence of Lfcin B at 0, 50, 100, or $200 \mu \mathrm{g} / \mathrm{mL}$ for 6 , 12,18 , and $36 \mathrm{~h}$. After incubation, cell proteins were separated using $8 \%$ SDS-PAGE. After transferring and blocking, cells were reacted with primary antibodies at $4^{\circ} \mathrm{C}$ overnight. Target proteins were subsequently detected using HRP-conjugated IgG with ECL plus and a Microtek Scanner (Microtek, Shanghai, China). Changes in DNMT1 protein content were detected by Western blotting using a DNMT1-specific antibody. Treatment with Lfcin B progressively reduced DNMT1 protein levels at 12, 18, and $36 \mathrm{~h}$ (Figure 2). 


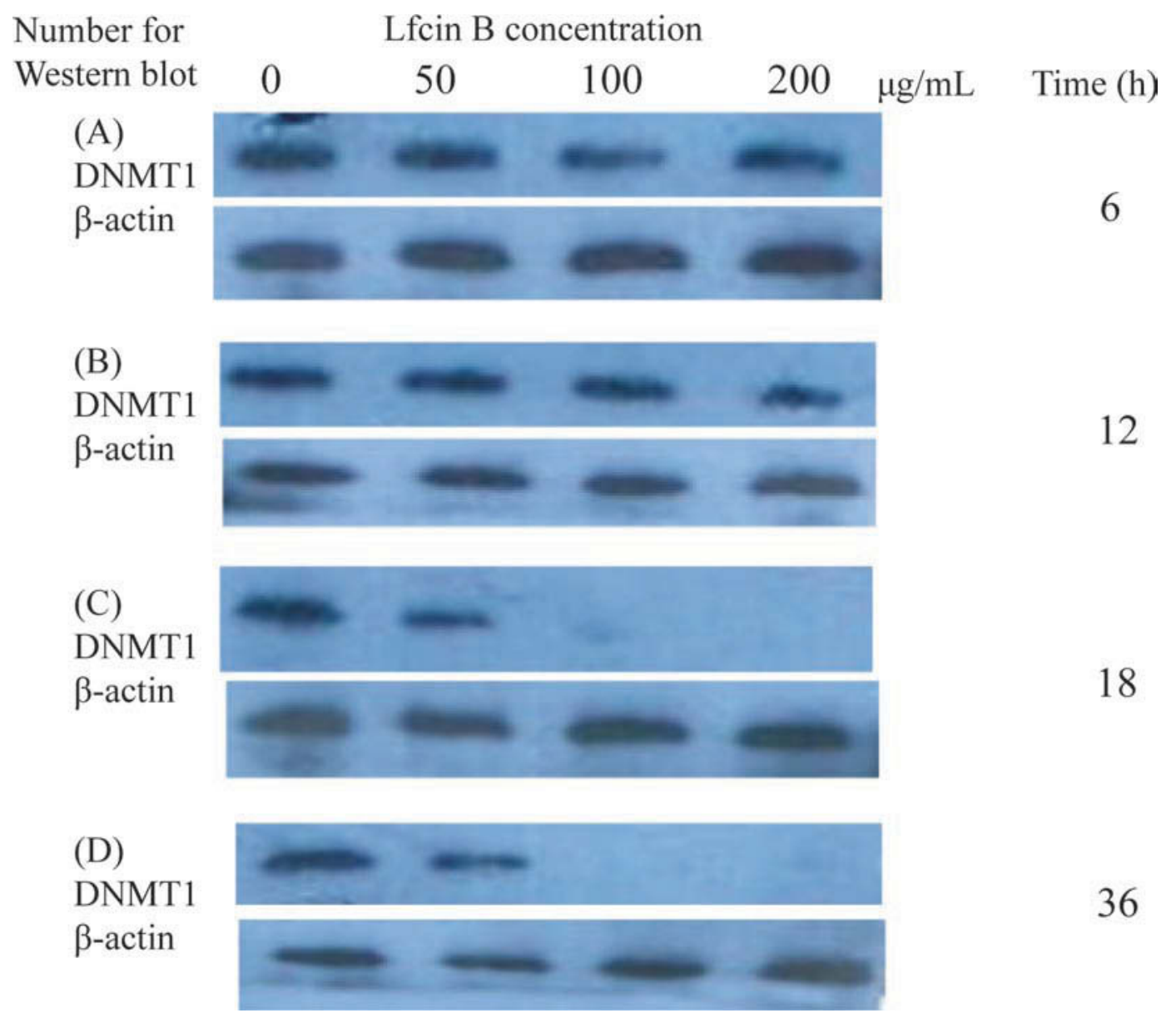

Figure 2. Down-modulation of DNA methyltransferase (DNMT)1 protein expression in Jurkat T-leukemia cells by lactoferricin B (Lfcin B). Jurkat T-leukemia cells were incubated in the presence of Lfcin B at 0, 50, 100, and $200 \mu \mathrm{g} / \mathrm{mL}$ for 6 (A), 12 (B), 18 (C), and 36 (D) h. The experiment was repeated 3 times. Color version available in online PDF.

\section{Lfcin B Reduces the Stability and Half-Life of DNMT1 mRNA}

The half-life of DNMT1 mRNA in Jurkat T-leukemia cells in the absence or presence of Lfcin B was compared by qRT-PCR. We found that Lfcin B decreased the stability of DNMT1 mRNA and reduced its half-life from approximately 8 to $2 \mathrm{~h}$ (Figure 3 ).

\section{Protein Synthesis Is Involved in the Destabilization of DNMT1 mRNA by Lfcin B}

Cells were divided into 3 groups, each containing 3 samples. Figure 4 shows that the relative expression level of DNMT1 in group 1, in which Jurkat T-leukemia cells were cultured for $6 \mathrm{~h}$ and then blocked with actinomycin $\mathrm{D}$ for $8 \mathrm{~h}$, was $28.12 \%$. The relative expression level of DNMT1 in group 2, in which Jurkat T-leukemia cells were cultured for $2 \mathrm{~h}$, Lfcin B was added and incubated for $4 \mathrm{~h}$, and then blocked with actinomycin $\mathrm{D}$ for
8 h, was $15.19 \%$. Relative expression level of DNMT1 in group 3 , in which the cells were blocked for $2 \mathrm{~h}$ with $10 \mu \mathrm{g} / \mathrm{mL}$ cycloheximide (a protein synthesis inhibitor), Lfcin B was added and incubated for $4 \mathrm{~h}$, and then blocked with actinomycin D for $8 \mathrm{~h}$, was $62.43 \%$. These results indicate that protein synthesis in Jurkat T-leukemia cells was necessary for Lfcin B to decrease the stability of DNMT1 mRNA (Figure 4).

\section{DISCUSSION}

Leukemia is a malignant disease caused by an imbalance between proliferation and maturation of blood cells or hematopoietic cells, although the precise mechanism remains unknown. Many of the altered properties of malignant cells can be attributed to genetic changes in critical genes, but it is now clear that epigenetic changes are also widespread in leukemia. Genetic changes include point mutations, gene deletions, and rearrangements, whereas epigenetic changes can tem- 


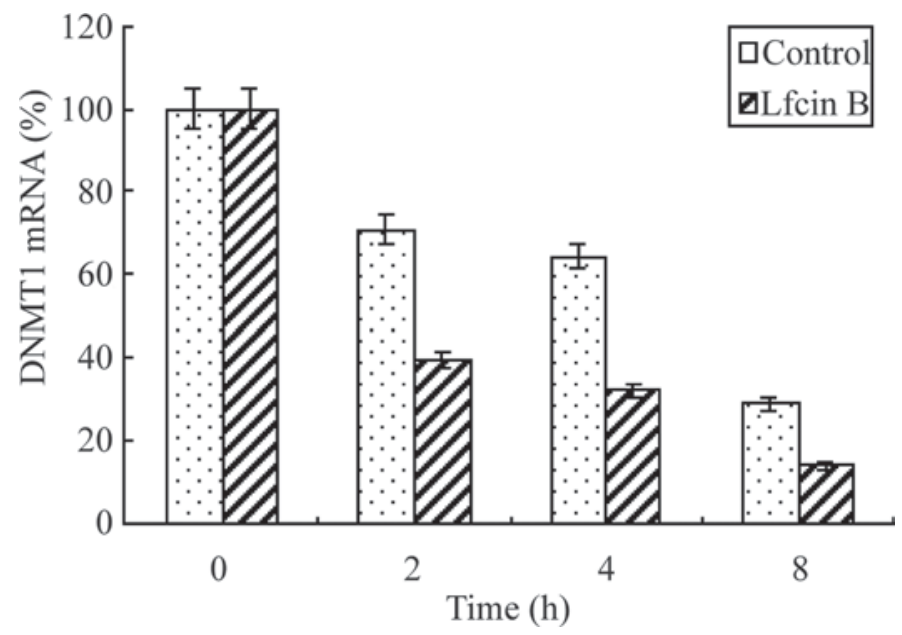

Figure 3. Rapid degradation of DNA methyltransferase (DNMT)1 mRNA in Jurkat T-leukemia cells induced by lactoferricin B (Lfcin B). Treatment with Lfcin B significantly $(P<0.05)$ reduced DNMT1 mRNA stability. Control = actinomycin D alone. Three samples were measured from each group and the results represent means \pm SE of the 3 groups.

porally and spatially control gene expression without changing the DNA sequence. Cytosine methylation at $\mathrm{CpG}$ dinucleotides is the most widely researched epigenetic change in mammals. Normal tissue-specific methylation patterns are established early in mammalian development, mediated by a combination of demethylation and de novo methylation (Baylin et al., 2001). Roman-Gomez et al. (2004) have shown that

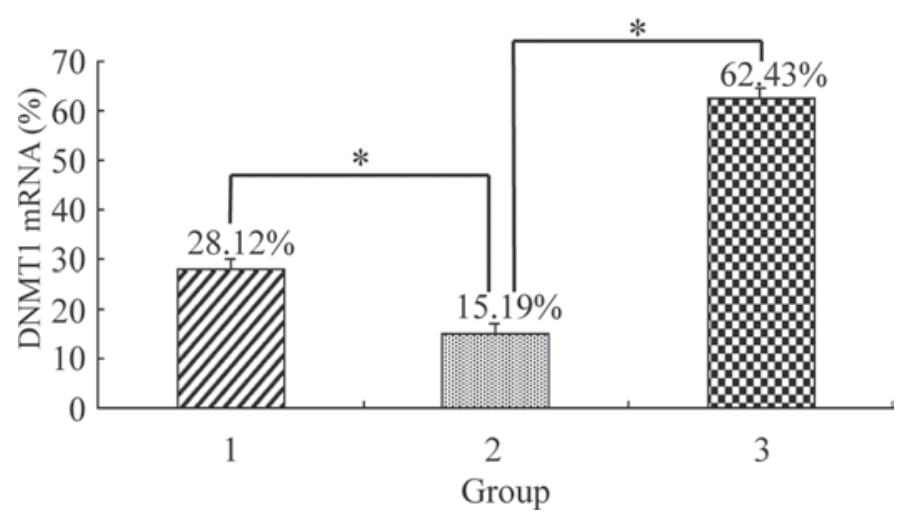

Figure 4. Protein synthesis in Jurkat T-leukemia cells plays a role in lactoferricin B (Lfcin B)-mediated DNA methyltransferase $($ DNMT) 1 mRNA destabilization. Control = Jurkat T-leukemia cells cultured for $14 \mathrm{~h}$; group $1=$ cells were cultured for $6 \mathrm{~h}$ then blocked with actinomycin D for 8 h; group 2 = cells were cultured for $2 \mathrm{~h}$, treated with Lfcin B for $4 \mathrm{~h}$, and then blocked with actinomycin D for $8 \mathrm{~h}$; group $3=$ cells were blocked for $2 \mathrm{~h}$ with $10 \mu \mathrm{g} / \mathrm{mL}$ cycloheximide (a protein synthesis inhibitor), treated with Lfcin B for $4 \mathrm{~h}$, and then blocked with actinomycin D for $8 \mathrm{~h}$. Real-time quantitative reverse transcription PCR results were expressed as percentages of their respective controls. Three samples were measured from each group and the results represent means $\pm \mathrm{SE}$ of the 3 groups; ${ }^{*} P<0.05$. aberrant methylation of $\mathrm{CpG}$ islands is quantitatively different in individual tumors within the same tumor type and that this patient-specific methylation profile provides important prognostic information in patients with acute lymphoblastic leukemia.

Although Lfcin B has attracted considerable interest because of its antimicrobial activity (Yamauchi et al., 1993), recent evidence indicates that Lfcin B also possesses potent in vivo activity against cancer cells. Roy et al. (2002) found that Lfcin B inhibited the proliferation of human leukemic cells (HL-60). Mader et al. (2005) observed that Lfcin B induced apoptosis in Jurkat Tleukemia cells and caused DNA fragmentation, nuclear condensation, and poly ADP-ribose polymerase cleavage. Apoptosis induction was triggered by a sequence of events comprising Lfcin B-mediated permeabilization of the cell membrane, Lfcin B aggregation on the sides of mitochondria, and subsequent depolarization of the mitochondrial membrane. This led to the release of cytochrome $\mathrm{C}$ and initiation of the intrinsic pathway of apoptosis (Mader et al., 2007). They also discovered that modulation of ceramide metabolism in Jurkat T-leukemia cells was involved in the induction of apoptosis induced by Lfcin B (Furlong et al., 2008). Freiburghaus et al. (2009) showed that Lfcin B treatment significantly, albeit slightly, prolonged the S phase in $\mathrm{CaCo}-2$ cells.

However, the effect of bovine lactoferricin on DNMT1 levels in Jurkat T-leukemia cells is not completely understood. In the current study, Lfcin B decreased the level of DNMT1 mRNA in Jurkat T-leukemia cells. Western blot analysis revealed a reduction of DNMT1 protein expression in cells. Our results indicated that activity of Lfcin B reduced the stability of DNMT1 mRNA, and this activity could be reduced by protein synthesis in Jurkat T-leukemia cells. These data support our hypothesis that Lfcin B might affect the expression of DNMT and the stability of DNMT mRNA in Jurkat T-leukemia cells. The cytotoxic activity of Lfcin B in human cancer cell lines suggests that it could be useful for the treatment of certain human cancers.

\section{ACKNOWLEDGMENTS}

Supported by grant No. CXT007-3-1 from the Innovative Team of Developmental Science and Technology of Bio-Dairy Products, Northeast Agricultural University (Harbin, China).

\section{REFERENCES}

Bartolomei, M. S., and S. M. Tilghman. 1997. Genomic imprinting in mammals. Annu. Rev. Genet. 31:493-525.

Baylin, S. B., M. Esteller, M. R. Rountree, K. E. Bachman, K. Schuebel, and J. G. Herman. 2001. Aberrant patterns of DNA 
methylation, chromatin formation and gene expression in cancer. Hum. Mol. Genet. 10:687-692.

Bellamy, W., M. Takase, H. Wakabayashi, K. Kawase, and M. Tomita. 1992a. Antibacterial spectrum of lactoferricin B, a potent bactericidal peptide derived from the N-terminal region of bovine lactoferrin . J. Appl. Bacteriol. 73:472-479.

Bellamy, W., M. Takase, K. Yamauchi, H. Wakabayashi, K. Kawase, and K. Tomita. 1992b. Identification of the bactericidal domain of lactoferrin. BBA-Prot. Proteom. 1121:130-136.

Cho, E., S. A. Smith-Warner, D. Spiegelman, W. L. Beeson, P. A. van den Brandt, G. A. Colditz, A. R. Folsom, G. E. Fraser, J. L. Freudenheim, E. Giovannucci, R. A. Goldbohm, S. Graham, A. B. Miller, P. Pietinen, J. D. Potter, T. E. Rohan, P. Terry, P. Toniolo, M. J. Virtanen, W. C. Willett, A. Wolk, K. Wu, S. S. Yaun, A Zeleniuch-Jacquotte, and D. J. Hunter. 2004. Dairy foods, calcium, and colorectal cancer: A pooled analysis of 10 cohort studies. J. Natl. Cancer Inst. 96:1015-1022.

Eliassen, L. T., G. Berge, M. Leknessund A Wikman, I. Lindin, C. Løkke, F. Ponthan, J. I. Johnsen, B. Sveinbjørnsson, P. Kogner, T. Flægstad, and $\varnothing$. Rekdal. 2006. The antimicrobial peptide, Lactoferricin B, is cytotoxic to neuroblastoma cells in vitro and inhibits xenograft growth in vivo. Int. J. Cancer 119:493-500.

Eliassen, L. T., G. Berge, B. Sveinbjørnsson, J. S. Svendsen, L. H. Vorland, and Ø. Rekdal. 2002. Evidence for a direct antitumor mechanism of action of bovine lactoferricin. Anticancer Res. 22:2703-2710.

Enrique, M., P. Manzanares, M. Yuste, M. Martínez, S. Vallés, and J. F. Marcos. 2009. Selectivity and antimicrobial action of bovine lactoferrin derived peptides against wine lactic acid bacteria. Food Microbiol. 26:340-346.

Freiburghaus, C., B. Janicke, H. Lindmark-Månsson, S. M. Oredsson, and M. A. Paulsson. 2009. Lactoferricin treatment decreases the rate of cell proliferation of a human colon cancer cell line. J. Dairy Sci. 92:2477-2484.

Furlong, S. J., N. Ridgway, and D. W. Hoskin. 2008. Modulation of ceramide metabolism in T-leukemia cell lines potentiates apoptosis induced by the cationic antimicrobial peptide bovine lactoferricin. Int. J. Oncol. 32:537-544.

Herman, J. G., C. I. Civin, J. P. Issa, M. I. Collector, S. J. Sharkis, and S. B. Baylin. 1997. Distinct patterns of inactivation of p15INK4B and p16INK4A characterize the major types of hematological malignancies. Cancer Res. 57:837-841.

Herman, J. G., J. Jen, A. Merlo, and S. B. Baylin. 1996. Hypermethylation-associated inactivation indicates a tumor suppressor role for p15INK4B. Cancer Res. 56:722-727.

Kass, S. U., D. Pruss, and A. P. Wolffe. 1997. How does DNA methylation repress transcription? Trends Genet. 13:444-449.

Kuwata, H., T. T. Yip, M. Tomita, and T. W. Hutchens. 1998. Direct evidence of the generation in human stomach of an antimicrobial peptide domain (lactoferricin) from ingested lactoferrin. Biochim. Biophys. Acta 1429:129-141.

Li, E., T. H. Bestor, and R. Jaenisch. 1992. Targeted mutation of the DNA methyltransferase gene results in embryonic lethality. Cell 69:915-926.
Livak, K. J., and T. D. Schmittgen. 2001. Analysis of relative gene expression data using real-time quantitative PCR and the 2(-delta delta $\mathrm{C}(\mathrm{T})$ ) method . Methods 25:402-408.

Lonnerdal, B., and S. Iyer. 1995. Lactoferrin: Molecular structure and biological function. Annu. Rev. Nutr. 15:93-110.

Mader, J. S., A. Richardson, J. Salsman, D. Top, R. de Antueno, R. Duncan, and D. W. Hoskin. 2007. Bovine lactoferricin causes apoptosis in Jurkat T-leukemia cells by sequential permeabilization of the cell membrane and targeting of mitochondria. Exp. Cell Res. 313:2634-2650.

Mader, J. S. J. Salsman, D. M. Conrad, and D. W. Hoskin. 2005. Bovine lactoferricin selectively induces apoptosis in human leukemia and carcinoma cell lines. Mol. Cancer Ther. 4:612-624.

Mizuno, S., T. Chijiwa, T. Okamura, K. Akashi, Y. Fukumaki, Y. Niho, and H. Sasaki. 2001. Expression of DNA methyltransferases DNMT1, 3A, and 3B in normal hematopoiesis and in acute and chronic myelogenous leukemia. Blood 97:1172-1179.

Nakao, M., and H. Sasaki. 1996. Genomic imprinting: significance in development and diseases and the molecular mechanisms. J. Biochem. 120:467-473.

Panning, B., and R. Jaenisch. 1998. RNA and the epigenetic regulation of X chromosome inactivation. Cell 93:305-308.

Robertson, K. D. 2002. DNA methylation and chromatin. Oncogene 21:5361-5379.

Roman-Gomez, J., A. Jimenez-Velasco, J. A. Castillejo, X. Agirre, M. Barrios, G. Navarro, F. J. Molina, M. J. Calasanz, F. Prosper, A. Heiniger, and A. Torres. 2004. Promoter hypermethylation of cancer-related genes: a strong independent prognostic factor in acute lymphoblastic leukemia. Blood 104:2492-2498.

Roy, M. K., Y. Kuwabara, K. Hara, Y. Watanabe, and Y. Tamai. 2002. Peptides from the n-terminal end of bovine lactoferrin induce apoptosis in human leukemic (HL-60) cells. J. Dairy Sci. 85:2065-2074.

Singal, R., and D. G. Ginder. 1999. DNA methylation. Blood 93:4059-4070.

Tomita, M., W. Bellamy, M. Takase, K. Yamauchi, H. Wakabayashi, and K. Kawase. 1991. Potent antibacterial peptides generated by pepsin digestion of bovine lactoferrin. J. Dairy Sci. 74:41374142

Tsuda, H., K. Sekine, and J. Nakamura. 1998. Inhibition of azoxymethane initiated colon tumor and aberrant crypt foci development by bovine lactoferrin administration in F344 rats. Adv. Exp. Med. Biol. 443:273-284.

Vogel, H. J., D. J. Schibli, W. Jing, E. M. Lohmeier-Vogel, R. F. Epand, and R. M. Epand. 2002. Towards a structure-function analysis of bovine lactoferricin and related tryptophan- and arginine-containing peptides. Biochem. Cell Biol. 80:49-63.

Yamauchi, K., M. Tomita, T. J. Giehl, and R. T. Ellison III.. 1993. Antibacterial activity of lactoferrin and a pepsin-derived lactoferrin peptide fragment. Infect. Immun. 61:719-728.

Yoo, Y. C., S. Watanabe, R. Watanabe, K. Hata, K. Shimazaki, and I. Azuma. 1997. Bovine lactoferrin and lactoferricin, a peptide derived from bovine lactoferrin, inhibit tumor metastasis in mice. Jpn. J. Cancer Res. 88:184-190. 